\title{
Oral Health Conditions and Treatment Needs of Athletes in Riyadh, Kingdom of Saudi Arabia
}

\author{
Nour AHM BinSalem ${ }^{1}$, Navin A Ingle ${ }^{2}$, Mohammad A Baseer ${ }^{3}$, Jamal A Alsanea ${ }^{4}$, Mansour K Assery ${ }^{5}$, Asma H Almeslet ${ }^{6}$
}

\begin{abstract}
Introduction: Oral health is integral to general health. Poor oral health has negative impact on oral health-related quality of life, thereby affecting athlete's performance and training.

Aims and objectives: To determine the oral health conditions and treatment requirements amongst athletes by examining the dental records at Prince Faisal Bin Fahad Bin Abdulaziz Sports Medicine Hospital, Riyadh, Kingdom of Saudi Arabia.

Materials and methods: A descriptive study based on the oral health records of athletes seeking care at Prince Faisal Bin Fahad Bin Abdulaziz Sports Medicine Hospital, Riyadh, Kingdom of Saudi Arabia, was carried out. Data of 331 athletes who underwent oral health screening in the past 5 years (January 2014-December 2018) within the dental department of Prince Faisal Bin Fahad Bin Abdulaziz Sports Medicine Hospital, Riyadh, Kingdom of Saudi Arabia, were obtained and analyzed for oral health conditions and treatment needs of the athletes. Descriptive statistics, Chi-square tests, Mann-Whitney, and Kruskal-Wallis tests were applied to the data to compare variables.

Results: Finding revealed 235 (71\%) tooth decay and 101 (31.5\%) need for teeth extraction among athletes. The mean ranks of extracted teeth were higher in the age group of 30-35 years (206.72) followed by 26-30 years (166.15), 15-20 years (165.79), and 21-25 years (155.19) with significant difference ( $\mathrm{KWH}=7.928, \mathrm{df}=3, p=0.048)$. Athletes aged 30-35 years (137.37) demonstrated significantly higher mean ranks of teeth extractions compared to $15-20$ years (109.65) (MW- $U=654.500, Z=-2.817, p=0.005)$. Similarly, athletes aged 30-35 years (66.54) demonstrated significantly higher mean ranks of teeth extractions compared to $21-25$ years (49.89) (MW- $U=1739.500, Z=-2.343, p=0.005)$. Gingival status showed significant differences across various age groups of the athletes (Chi-square $=8.375, \mathrm{df}=3, p=0.039$ )

Conclusion: Data from Prince Faisal Bin Fahad Bin Abdulaziz Sports Medicine Hospital, Riyadh, Kingdom of Saudi Arabia, suggest that the oral health of the athletes needs improvement especially with regards to the dental caries, teeth extractions, and periodontal conditions. Hence, oral health promotional and preventive programs should be initiated to improve oral health of the athletes.

Keywords: Athletes, Dental caries, Extraction, Oral health, Periodontal disease, Scaling, Treatment needs.

Journal of Oral Health and Community Dentistry (2019): 10.5005/.jp-journals-10062-0054
\end{abstract}

\section{INTRODUCTION}

Oral health is considered as one of the important concerns among athletes because of their likelihood of having trauma and oral diseases such as dental caries, erosion, and periodontal disease. ${ }^{1,2}$ There are numerous factors that causes athletes to become susceptible to oral health diseases such as the consumption of energy drinks, irregular toothbrushing and flossing, individual biological setup, physical and socioeconomic environment, personal behaviors and lifestyle, and availability of organized healthcare services. ${ }^{3}$

Oral health is strongly associated with diet in various manners. Nutrition can affect the growth and development of craniofacial tissues and there could be susceptibility for the development of oral infectious diseases. These include dental caries, pulpitis, gingivitis, and periodontitis. Moreover, oral health is affected by bruxism, traumatic dental injuries, and abnormal bite. ${ }^{4}$ Intake of the carbohydrate along with the acidic foods and beverages is one of the important factors in causing dental caries and dental erosion. Moreover, in order to decrease the rate of dental erosion, ingestion of carbohydrate, fruit drinks, sports drinks, vinegar, citrus fruits, and berries should be restricted. ${ }^{5}$

Facial appearance is important for the athletes, as tooth loss can have embarrassing situations specifically during the speech. Such situation is not accepted by the athletes. ${ }^{6}$ Oral health is an important element of the quality of life. Poor oral health is associated with pain, unsightly appearance, and strong effects on confidence and quality
${ }^{1}$ Department of Advanced Education in General Dentistry, College of Dentistry, Riyadh Elm University, Riyadh, Kingdom of Saudi Arabia

${ }^{2}$ Department of Dental Public Health, College of Dentistry, Riyadh Elm University, Riyadh, Kingdom of Saudi Arabia

${ }^{3-5}$ Department of Preventive Dentistry, College of Dentistry, Riyadh Elm University, Riyadh, Kingdom of Saudi Arabia

${ }^{6}$ Department of Oral Maxillofacial Surgery and Diagnostic Sciences, College of Dentistry, Riyadh Elm University, Riyadh, Kingdom of Saudi Arabia

Corresponding Author: Nour AHM BinSalem, Department of Advanced Education in General Dentistry, College of Dentistry, Riyadh Elm University, Riyadh, Kingdom of Saudi Arabia, e-mail: nour.a.binsalem@student.riyadh.edu.sa

How to cite this article: BinSalem NAHM, Ingle NA, Baseer MA, et al. Oral Health Conditions and Treatment Needs of Athletes in Riyadh, Kingdom of Saudi Arabia. J Oral Health Comm Dent 2019;13(3):84-89. Source of support: Nil

Conflict of interest: None

of life and may have burden of accumulated dental treatment needs leading to lowered self-esteem. ${ }^{7}$

The oral health of the athletes faces a variety of challenges ranging from nutritional conditions, oral dehydration, exercisedriven immune suppression, lack of awareness, negative health behaviors to less priority to the oral health. Moreover, due to the 
inadequate oral health literacy, athletes more likely to obtain examination and treatments during oral health problems and to seek less regular dental care. ${ }^{8}$

Information on oral health status of athletes is essential for various reasons. First of all, it is important to know the magnitude of oral health problems among athletes in order to develop and implement the oral health resources and services. Second, it is important to determine the need for preventive oral care for the athletes. Oral health is integral to general health. Poor oral health has negative impact on oral health-related quality of life, thereby affecting athletic performance and training. ${ }^{1}$

Prince Faisal Bin Fahad Bin Abdulaziz Sports Medicine Hospital was established in 1985 in an attempt to cover the needs of the Saudi youth and to complement the several sports facilities within the Kingdom of Saudi Arabia. This is a center for providing distinctive services in all fields of sports medicine ranging from the diagnosis and treatment of sports injuries to the multifaceted professional specialty services. It provides specialized athlete's healthcare and prevention services including exercise physiology, biomechanics, sport psychology, and sports nutrition. It is also specialized in the field of clinical sports medicine in all its prevention and treatment aspects. This hospital has several departments including the dental department, providing needed healthcare services to the athletes. There is a lack of reported oral health data among athletes from Saudi Arabia. Hence, this study aimed to determine the oral health conditions and treatment requirements amongst athletes by examining the dental records at Prince Faisal Bin Fahad Bin Abdulaziz Sports Medicine Hospital, Riyadh.

\section{Materials and Methods}

\section{Ethical Approval}

The study proposal was submitted to the research center of Riyadh Elm University (FPGRP/437310011/280) and the Institutional Review Board approval was obtained (RC/IRB/2018/1269). The permission to access the dental records of the athletes was obtained from the Prince Faisal Bin Fahad Bin Abdulaziz Sports Medicine Hospital, Riyadh.

\section{Study Design}

This was a descriptive study based on the screened oral records of athletes seeking oral healthcare at Prince Faisal Bin Fahad Bin Abdulaziz Sports Medicine Hospital, Riyadh, Kingdom of Saudi Arabia. Data of athletes who underwent oral health screening in the past 5 years (January 2014-December 2018) within the dental department of Prince Faisal Bin Fahad Bin Abdulaziz Sports Medicine Hospital, Riyadh, Kingdom of Saudi Arabia, were obtained and analyzed for oral health conditions and treatment needs of the athletes.

\section{Contents of the Dental Screening Form}

Oral health screening form consisted of demographic information of age, gender, and nationality of the athletes. Examination part of the form included extraoral, intraoral, and dental examinations sections. A number of decayed and extracted teeth were also noted. Oral hygiene status, gingival, and periodontal conditions were recorded on a dichotomous (good or bad) scale. Treatment plan part recorded necessary treatments of the conditions.

\section{Sample Size}

A priori analysis was performed to compute required sample size by using $G^{*}$ power software by considering a effect size of 0.228 , an alpha $(\alpha)$ error probability of 0.05 , a power of the study (1- $\beta$ error probability) of 0.95 , and a degree of freedom (df) of 3 . The required sample size was found to be 331 . All the forms were collected by employing convenient sampling methodology from single sports medicine hospital.

\section{Obtaining Screening Forms}

Total of 331 oral health screening forms of athletes were retrieved from the dental records department. A general dental practitioner serving within the hospital collected the forms. All the variables in the screening form were coded and entered into the excel sheet by the investigator. Later this information was transferred to the special statistical analysis software for further analysis.

\section{Statistical Analysis}

Data were checked for the normality by applying Shapiro-Wilk and Kolmogorov-Smirnov tests and data were found to be not normally distributed $(p<0.05)$. Descriptive statistics of frequency distribution and percentages were reported for the categorical variables while mean and standard deviation values were calculated for the continuous variables. Chi-square tests were applied to assess the relationship between categorical variables. Mean ranks of decayed and extracted teeth were compared across different age groups and nationalities by applying Kruskal-Wallis and Mann-Whitney $U$ tests. A $p$ value $(p<0.05)$ was considered significant for all statistical purposes. All the statistical analysis was performed using IBM-SPSS Version 21 software, Armonk: NY, USA.

\section{Results}

A total of 331 dental screening records pertaining to the athletes were assessed for their oral health conditions. Most of the athletes, 201 (60.7\%) were aged between 15 years and 20 years followed by other age groups. All the records were pertained to the male and most, 324 (97.9\%), of them were Saudi nationals. Tooth decay 235 (71\%) and need for teeth extraction of 101 (31.5\%) were observed among athletes. Most of the study participants had good oral hygiene (65.3\%), gingival (69.5\%), and periodontal conditions (87\%). Dental abnormalities were observed in $6(1.8 \%)$ athletes while need for dental scaling was found in 247 (74.6\%) athletes (Table 1).

The presence of decayed and extracted teeth was compared across the different age groups. Athletes aged 15-20 years had the mean rank of decayed teeth (168.03) followed by 21-25 years (154.20), 26-30 years (149.88), and 30-35 years (207.67). There was no significant difference in the mean ranks of decayed teeth $(\mathrm{KWH}=$ $6.631, \mathrm{df}=3, p=0.085$ ), while mean ranks of extracted teeth were higher in age group of 30-35 years (206.72) followed by 26-30 years (166.15), 15-20 years (165.79), and 21-25 years (155.19) with significant difference ( $\mathrm{KWH}=7.928, \mathrm{df}=3, p=0.048$ ), as shown in Table 2.

Further, athletes aged 30-35 years (137.37) demonstrated significantly higher mean ranks of teeth extractions compared to $15-20$ years (109.65) ( $\mathrm{MW}-U=654.500, Z=-2.817, p=0.005)$. Similarly, athletes aged $30-35$ years (66.54) demonstrated significantly higher mean ranks of teeth extractions compared to $21-25$ years (49.89) (MW- $U=1739.500, Z=-2.343, p=0.005)$, as shown in Table 3.

Saudi athletes exhibited higher mean ranks (166.46) of teeth extraction compared to non-Saudi athletes (144.71) without any statistically significant differences (MW- $U=985.000, Z=-0.733$ $p=0.464$ ) between them (Table 4). Similarly mean ranks of decayed 
teeth were higher among Saudi athletes (167.31) compared to the non-Saudis (105.21) without any statistically significant differences (MW-U = 708.500, $Z=-1.730, p=0.084$ ) (Table 4).

In general, oral hygiene status, gingival status, periodontal status, and reported dental abnormalities did not differ significantly between Saudi and non-Saudi athletes.

Poor gingival health was observed with $15-20$ years 56 $(27.9 \%), 21-25$ years $26(31.3 \%), 26-30$ years $6(25 \%)$, and

Table 1: Characteristics of the athletes $(n=331)$

\begin{tabular}{|c|c|c|c|}
\hline Characteristics & & $n$ & $\%$ \\
\hline \multirow[t]{4}{*}{ Age } & $15-20$ & 201 & 60.7 \\
\hline & $21-25$ & 83 & 25.1 \\
\hline & $26-30$ & 24 & 7.3 \\
\hline & $30-35$ & 23 & 6.9 \\
\hline \multirow[t]{2}{*}{ Gender } & Female & 0 & 0.0 \\
\hline & Male & 331 & 100.0 \\
\hline \multirow[t]{2}{*}{ Nationality } & Non-Saudi & 7 & 2.1 \\
\hline & Saudi & 324 & 97.9 \\
\hline \multirow[t]{2}{*}{ Tooth decay } & Present & 235 & 71 \\
\hline & Absent & 96 & 29 \\
\hline \multirow[t]{2}{*}{ Need for tooth extraction } & Present & 101 & 31.5 \\
\hline & Absent & 230 & 69.5 \\
\hline \multirow[t]{2}{*}{ Oral hygiene } & Bad & 115 & 34.7 \\
\hline & Good & 216 & 65.3 \\
\hline \multirow[t]{2}{*}{ Gingival condition } & Bad & 101 & 30.5 \\
\hline & Good & 230 & 69.5 \\
\hline \multirow[t]{2}{*}{ Periodontal condition } & Bad & 43 & 13.0 \\
\hline & Good & 288 & 87.0 \\
\hline \multirow[t]{2}{*}{ Dental abnormalities } & Present & 6 & 1.8 \\
\hline & Absent & 325 & 98.2 \\
\hline \multirow[t]{2}{*}{ Need for scaling } & Present & 247 & 74.6 \\
\hline & Absent & 84 & 25.4 \\
\hline
\end{tabular}

Table 2: Comparison of decayed and extracted teeth among different age groups

\begin{tabular}{llllll}
\hline Variables & Age (years) & Mean rank & KWH & $d f$ & pvalue \\
\hline Decayed & $15-20$ & 168.03 & 6.631 & 3 & 0.085 \\
& $21-25$ & 154.20 & & & \\
& $26-30$ & 149.88 & & & \\
& $30-35$ & 207.67 & & & \\
Extraction & $15-20$ & 165.79 & 7.928 & 3 & 0.048 \\
& $21-25$ & 155.19 & & & \\
& $26-30$ & 166.15 & & & \\
& $30-35$ & 206.72 & & & \\
\hline
\end{tabular}

$\mathrm{KWH}$, Kruskal-Wallis $\mathrm{H}$; df, degrees of freedom
30-35 years 13 (56.5\%), while good gingival status was found in $15-20$ years $145(72.1 \%), 21-25$ years $57(68.7 \%), 26-30$ years 18 (75\%), and $30-35$ years 10 (43.5\%). Gingival status showed significant differences across various age groups of the athletes (Chi-square $=8.375, \mathrm{df}=3, p=0.039$ ). However, periodontal status and dental abnormalities were recorded without any significant differences across age groups as shown in Table 5.

More than half $(57.1 \%)$ of the non-Saudi athletes and threefourths $(75 \%)$ of the Saudi athletes were in need of scaling to remove dental calculus deposits without any significant differences between them (Chi-square $=1.154$, df $=1, p=0.283$ ). Similarly, no significant differences were observed with regards to the age groups and need for the scaling among athletes (Chi-square $=4.286$, $\mathrm{df}=3, p=0.232$ ) as shown in Table 6.

\section{Discussion}

There is a lack of oral health information among athletes from Saudi Arabia. Therefore, this study reports the oral health conditions and treatment requirements of the athletes by retrospective analysis of screening data from dental department of Prince Faisal Bin Fahad Bin Abdulaziz Sports Medicine Hospital, Riyadh. High prevalence of decayed teeth and need for extraction of teeth is suggestive of poor oral health conditions among athletes. This finding is consistent with the other reported studies in which soccer players demonstrated poor oral health status. ${ }^{9,10}$ The mean decayed missing filled teeth (DMFT) of professional soccer players was found to be higher than that of the adults as reported by national survey in Thailand. ${ }^{10}$ Similarly, findings of higher DMFT scores were reported by Barcelonan and Spanish studies when compared to the students. ${ }^{11}$ In our study, only presence and absence of decayed teeth among athletes have been recorded to know the active disease process. Moreover, teeth indicated

Table 3: Comparison of mean ranks of extracted teeth in different age groups

\begin{tabular}{llllll}
\hline Age & Mean rank & Sum of ranks & $M W-U$ & $Z$ & $p$ value \\
\hline $15-20$ & 145.16 & 29177.50 & 7806.500 & -1.067 & 0.286 \\
$21-25$ & 136.05 & 11292.50 & & & \\
$15-20$ & 112.97 & 22707.50 & 2406.500 & -0.023 & 0.982 \\
$26-30$ & 113.23 & 2717.50 & & & \\
$15-20$ & 109.65 & 22040.50 & 1739.500 & -2.343 & 0.019 \\
$30-35$ & 137.37 & 3159.50 & & & \\
$21-25$ & 53.25 & 4419.50 & 933.500 & -0.606 & 0.544 \\
$26-30$ & 56.60 & 1358.50 & & & \\
$21-25$ & 49.89 & 4140.50 & 654.500 & -2.817 & 0.005 \\
$30-35$ & 66.54 & 1530.50 & & & \\
$26-30$ & 21.31 & 511.50 & 211.500 & -1.552 & 0.121 \\
$30-35$ & 26.80 & 616.50 & & & \\
\hline
\end{tabular}

MW-U, Mann-Whitney $U$ test

Table 4: Mean ranks of extracted and decayed teeth based on nationality of athletes

\begin{tabular}{lllclll}
\hline Variables & Nationality & Mean rank & Sum of ranks & MW-U & $Z$ & $p$ value \\
\hline Extraction & Non-Saudi & 144.71 & 1013.00 & 985.000 & -0.733 & 0.464 \\
& Saudi & 166.46 & 53933.00 & & & \\
\multirow{2}{*}{ Decayed } & Non-Saudi & 105.21 & 736.50 & 708.500 & -1.730 & 0.084 \\
& Saudi & 167.31 & 54209.50 & & & \\
\hline
\end{tabular}

MW-U, Mann-Whitney $U$ test 


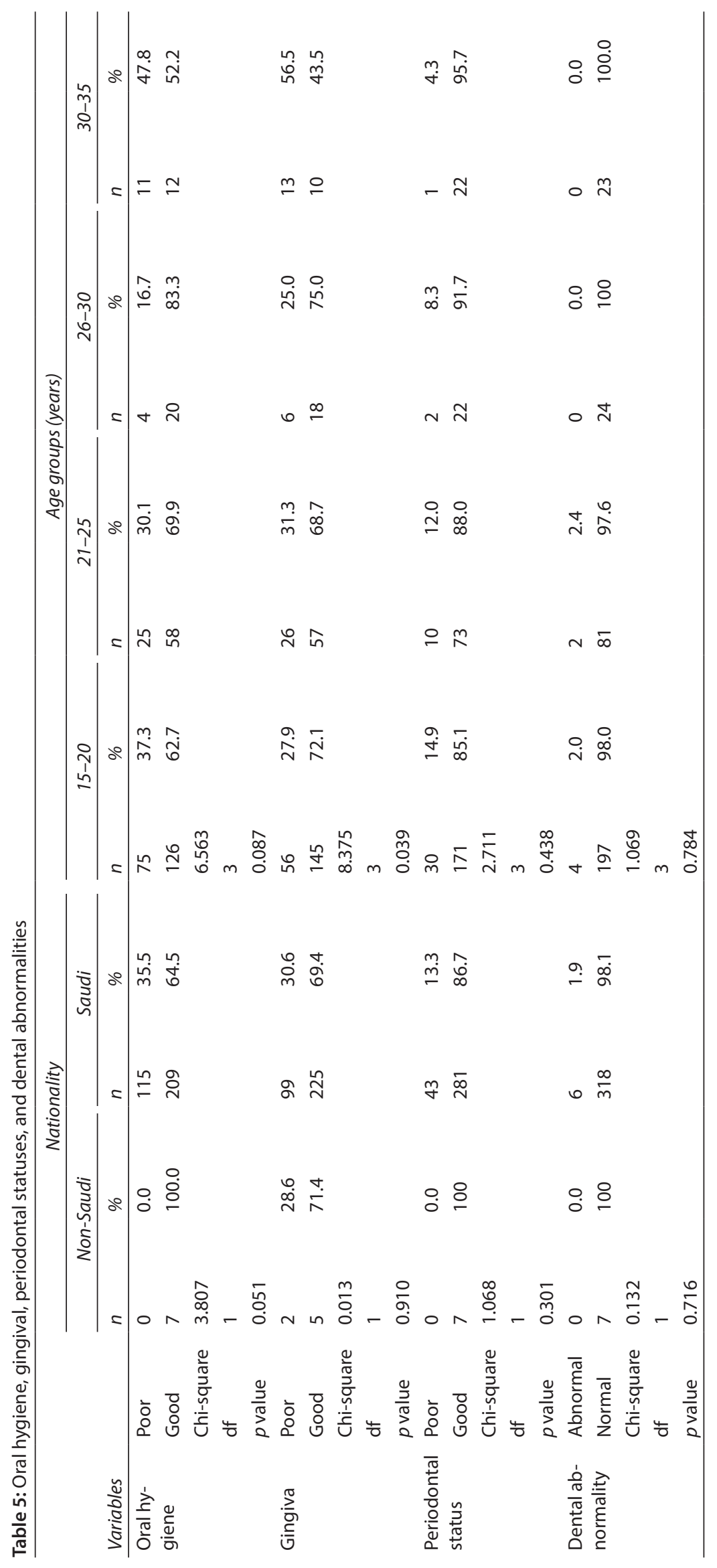


Oral Health Conditions and Treatment Needs of Athletes

Table 6: Scaling needs across nationality and age groups of the athletes

\begin{tabular}{|c|c|c|c|c|c|c|}
\hline \multirow[b]{2}{*}{ Scaling } & \multicolumn{2}{|c|}{ Nationality } & \multicolumn{4}{|c|}{ Age groups (years) } \\
\hline & Non-Saudi & Saudi & $15-20$ & $21-25$ & $26-30$ & $30-35$ \\
\hline No & $3(42.9 \%)$ & $81(25 \%)$ & $53(26.4 \%)$ & $21(25.3 \%)$ & $8(33.3 \%)$ & $2(8.7 \%)$ \\
\hline Yes & $4(57.1 \%)$ & $243(75 \%)$ & $148(73.6 \%)$ & $62(74.7 \%)$ & $16(66.7 \%)$ & $21(91.3 \%)$ \\
\hline Chi-square & 1.154 & & 4.286 & & & \\
\hline df & 1 & & 3 & & & \\
\hline$p$ value & 0.283 & & 0.232 & & & \\
\hline
\end{tabular}

for extraction irrespective of their periodontal and dental caries statuses were recorded in this study. The significant differences in need of extraction of teeth among the age groups of 30-35 years compared to the lower age groups point out toward oral health negligence among athletes.

Poor oral hygiene is a risk factor for all the oral diseases. ${ }^{12}$ Previous reports have shown that athletes brushed their teeth twice a day but their oral hygiene was still poor as evidenced by high plaque score and with gingivitis. Nearly $15-30 \%$ of players had periodontitis. ${ }^{2,10}$ Although the present study revealed that the most of the athletes had good oral hygiene, gingival, and periodontal conditions and still there were considerable percentages of athletes with poor oral health findings similar to the previously reported studies. Hence, there is a need for the oral health educational activities targeted toward the athletes.

Dental attrition common problem reported among athletes. Sports drinks have showed an association in causing dental attrition among athletes. ${ }^{10,13}$ It has been reported that sports drinks and reduced salivary flow at the time of exercise were associated with the lesions of tooth wear due to the loss of salivary protection against acid rather than by teeth clenching or toothbrush abrasion. ${ }^{14-17}$ This loss of salivary protection of the teeth is caused by work- and sport-related dehydration, drugs and certain medical condition, and syndromes. ${ }^{18}$ Another important area of concern among athletes is the occurrence of orofacial injuries. These injuries are mainly preventable by taking appropriate measures. However, studies have reported that most of the athletes were unaware about the implications of the traumatic injuries occurring on the orofacial region during playing. Injuries to the orofacial area could be effectively prevented by wearing mouthguards during the practice or competitive sessions. This will help to reduce the incidence of orofacial injuries among athletes. ${ }^{19,20}$ In this study, only few athletes had dental abnormalities compared to the previous studies. Dental scaling was the common treatment needed among athletes.

The strength of this study included the large number of screened files belonging to the athletes, which were studied from the dedicated sports hospital. Only oral health screening files with limited number of variables were utilized for the purpose of study. The athletes file belonging to a single center of Prince Faisal Bin Fahad Bin Abdulaziz Sports Medicine Hospitals was analyzed in this study. Hence, this study results are only applicable to the single center. These results may not reflect the current oral health situation of all the athletes in the Saudi Arabia. In order to gain complete understanding of the oral health conditions, an epidemiological study with a representative sample from multiple sports medicine hospitals scattered across Saudi Arabia is needed. Moreover, primary data on oral health should be collected based on the standard methods and forms prescribed by the WHO and other professional associations in order to assess the oral health status and treatment needs of the athletes.

\section{Conclusion}

Within the limitations of the study, it can be concluded that the screening data from Prince Faisal Bin Fahad Bin Abdulaziz Sports Medicine Hospital, Riyadh, suggests that the oral health of the athletes needs improvement especially with regards to the dental caries, teeth extractions, and periodontal conditions. Hence, oral health promotional programs followed by periodic examination and preventive treatments should be instituted to decrease oral health problems and to enable good oral health, well-being, and performance among athletes.

\section{References}

1. Ashley P, Di lorio A, Cole E, et al. Oral health of elite athletes and association with performance: a systematic review. $\mathrm{Br} J$ Sports Med 2015;49(1):14-19. DOI: 10.1136/bjsports-2014-093617.

2. Needleman I, Ashley P, Meehan L, et al. Poor oral health including active caries in 187 UK professional male football players: clinical dental examination performed by dentists. $\mathrm{Br} J$ Sports Med 2016;50(1):41-44. DOI: 10.1136/bjsports-2015-094953.

3. Rajapakse PS, McCracken GI, Gwynnett E, et al. Does tooth brushing influence the development and progression of noninflammatory gingival recession? A systematic review. J Clin Periodontol 2007;34(12):1046-1061. DOI: 10.1111/j.1600-051X.2007. 01149.x.

4. Sheiham A, Steele JG, Marcenes W, et al. The relationship among dental status, nutrient intake, and nutritional status in older people. J Dent Res 2001;80(2):408-413. DOI: 10.1177/00220345010800020201.

5. Baumgartner S, Imfeld T, Schicht O, et al. The impact of the stone age diet on gingival conditions in the absence of oral hygiene. J Periodontol 2009;80(5):759-768. DOI: 10.1902/jop.2009.080376.

6. Moynihan P, Petersen PE. Diet, nutrition and the prevention of dental diseases. Public Health Nutr 2004;7(1A):201-226. DOI: 10.1079/ PHN2003589.

7. Petersen $\mathrm{PE}$, Bourgeois $\mathrm{D}$, Ogawa $\mathrm{H}$, et al. The global burden of oral diseases and risks to oral health. Bull World Health Organ 2005;83(9):661-669. DOI: /S0042-96862005000900011.

8. McGovern LA, Spolarich AE, Keim R. A survey of attitudes, behaviors, and needs of team dentists. Gen Dent 2015;63(6):61-66.

9. Ljungberg $G$, Birkhed $D$. Dental caries in players belonging to a Swedish soccer team. Swed Dent J 1990;14(6):261-266.

10. Chantaramanee A, Samnieng P. Oral health status and impact on performance of professional soccer players. J Dent Indones 2016;23(1):1-4. DOI: 10.14693/jdi.v23i1.633.

11. Gay-Escoda C, Vieira-Duarte-Pereira D-M, Ardèvol J, et al. Study of the effect of oral health on physical condition of professional soccer players of the Football Club Barcelona. Med Oral Patol Oral Cirugia Bucal 2011;16(3):e436-e439. DOI: 10.4317/medoral.16.e436.

12. Solleveld H, Goedhart A, Vanden Bossche L. Associations between poor oral health and reinjuries in male elite soccer players: a crosssectional self-report study. BMC Sports Sci Med Rehabil 2015;7:11. DOI: 10.1186/s13102-015-0004-y.

13. Mathew T, Casamassimo PS, Hayes JR. Relationship between sports drinks and dental erosion in 304 university athletes in 
Columbus, Ohio, USA. Caries Res 2002;36(4):281-287. DOI: 10.1159/ 000063927.

14. Brouns F, Muntjewerf L. Sports drinks and teeth. Br J Sports Med 1997;31(3):258. DOI: 10.1136/bjsm.31.3.258.

15. Coombes JS. Sports drinks and dental erosion. Am J Dent 2005;18(2):101-104.

16. Mulic A, Tveit AB, Songe $D$, et al. Dental erosive wear and salivary flow rate in physically active young adults. BMC Oral Health 2012;12:8. DOI: 10.1186/1472-6831-12-8.
17. Broughton D, Fairchild RM, Morgan MZ. A survey of sports drinks consumption among adolescents. Br Dent J 2016;220(12):639-643. DOI: 10.1038/sj.bdj.2016.449.

18. Young WG. The oral medicine of tooth wear. Aust Dent J 2001;46(4):236-250. ; quiz 306 10.1111/j.1834-7819.2001.tb00288.x.

19. Flanders RA, Bhat M. The incidence of orofacial injuries in sports: a pilot study in Illinois. J Am Dent Assoc 1995;126(4):491-496. DOI: 10.14219/jada.archive.1995.0213.

20. Flanders RA. Project mouthguard. III Dent J 1995;64(2):67-69. 